\title{
Dependence of the Absorption-Line Profiles and Radial-Velocity Curve of the Optical Star in an X-ray Binary on the Orbital Inclination and Component-Mass Ratio
}

\author{
M. K. Abubekerov, É. A. Antokhina, and A. M. Cherepashchuk \\ Sternberg Astronomical Institute, Universitetskiu pr. 13, Moscow, 119992 Russia \\ Received December 18, 2004; in final form, May 18, 2005
}

\begin{abstract}
Theoretical absorption-line profiles and radial-velocity curves for tidally deformed optical stars in X-ray binary systems are calculated assuming LTE. The variations in the profile shapes and radialvelocity curve of the optical star are analyzed as a function of the orbital inclination of the X-ray binary system. The dependence of the shape of the radial-velocity curve on the orbital inclination $i$ increases with decreasing component-mass ratio $q=m_{x} / m_{v}$. The integrated line profiles and radial-velocity curves of the optical star are calculated for the Cyg X-1 binary, which are then used to estimate the orbital inclination and mass of the relativistic object: $i<43^{\circ}$ and $m_{x}=8.2-12.8 M_{\odot}$. These estimates are in good agreement with earlier results of fitting the radial-velocity curve of Cyg X-1 using a simpler model $\left(i<45^{\circ}\right.$, $\left.m_{x}=9.0-13.2 M_{\odot}\right)$. (C) 2005 Pleiades Publishing, Inc.
\end{abstract}

\section{INTRODUCTION}

The optical component in an X-ray binary system is tidally deformed and has a complex temperature distribution on its surface due to the effects of gravitational darkening and $\mathrm{X}$-ray heating. These effects of the interaction between the components give rise to orbital variability of the absorption-line profiles of an optical star. The orbital variability of the absorption profiles leads to a dependence of both the half-amplitude and the shape of the radial-velocity curve on the orbital inclination $i$ and the componentmass ratio $q=m_{x} / m_{v}$.

The dependence of the shape of the radial-velocity curve of a tidally deformed optical star on the parameters of a close binary system was first pointed out by Sofia and Wilson [1]. Antokhina and Cherepashchuk [2] and Shabaz [3] proposed a new method for determining the component-mass ratio $q=m_{x} / m_{v}$ and orbital inclination $i$ of an X-ray binary based on the orbital variability of the absorptionline profiles in the spectrum of the optical star. Abubekerov et al. [4] estimated the orbital inclination of the X-ray binary Cyg X-1 using a high-accuracy observational radial-velocity curve.

Here, we present the results of theoretical modeling of the radial-velocity curve of the optical star in a Roche model assuming LTE for various orbital inclinations, for X-ray systems with low-, moderate-, and high-mass optical stars. We analyze variations in the radial-velocity curve with variations of $i$ for various values of $q$, together with the corresponding variations in the $\mathrm{H} \gamma$ absorption-line profile.

\section{SYNTHESIS OF THE RADIAL-VELOCITY CURVES FOR THE OPTICAL STAR}

The synthesis of the theoretical absorption-line profiles and radial-velocity curves for the optical star in an X-ray binary system was carried out using the algorithm described in detail by Antokhina et al. $[5,6]$. We will briefly summarize the basis of this method here.

In the Roche model, the X-ray binary system consists of an optical star and a pointlike X-ray source. The star is tidally deformed and has a nonuniform surface-temperature distribution due to the effects of gravitational darkening and heating of the stellar surface by the X-ray emission of the relativistic object.

The surface of the optical star was divided into $\sim 2600$ area elements, for each of which we calculated the emergent local radiation assuming LTE. Each area element corresponds to a local temperature $T_{\text {loc }}$, local gravitational acceleration $g_{\text {loc }}$, and local value of the parameter $k_{x}^{\text {loc }}$, which is equal to the ratio of the incident $\mathrm{X}$-ray flux and the outgoing radiation flux without allowance for external irradiation of the atmosphere. Using these parameter values at a given point of the surface, a model for the atmosphere is calculated by solving the equation of line radiative transfer in the presence of incident external X-ray radiation. In this way, we can compute the intensity of the outgoing radiation in the line and continuum for each local area element. At different phases of the orbital period, the contributions of the areas to the total radiation are summed taking into account Doppler 
Table 1. Numerical values of parameters used to synthesize the radial-velocity curves of the optical component in the Roche model

\begin{tabular}{|c|c|c|}
\hline$P$, day & 5.0 & Period \\
\hline$m_{x}, M_{\odot}$ & 10 & Mass of the compact object \\
\hline$m_{v}, M_{\odot}$ & $v a r^{*}$ & Mass of the optical star \\
\hline$e$ & 0.0 & Eccentricity \\
\hline$i, \operatorname{deg}$ & $30,60,90$ & Orbital inclination \\
\hline$\mu$ & 1.0 & Roche lobe filling coefficient for the optical star \\
\hline$f$ & 1.0 & Asynchronicity coefficient for the rotation of the optical star \\
\hline$T_{\text {eff }}, \mathrm{K}$ & $v a r^{*}$ & Effective temperature of the optical star \\
\hline$\beta$ & $0.25^{* *}$ & Gravitational-darkening coefficient \\
\hline$k_{x}$ & 0.1 & $\begin{array}{l}\text { Ratio of the X-ray luminosity of the relativistic component to the } \\
\text { bolometric luminosity of the optical component } L_{x} / L_{v}\end{array}$ \\
\hline$A$ & 0.5 & Reprocessing coefficient for the X-ray radiation \\
\hline$u$ & 0.3 & Limb-darkening coefficient \\
\hline
\end{tabular}

* Parameter of the $\mathrm{X}$-ray binary was varied during the model computations.

** When the mass of the optical star was $m_{v}=1 M_{\odot}$, the gravitational-darkening coefficient was taken to be $\beta=0.08$.

effects and the conditions for visibility of the areas by an observer. This yields the total radiation flux from the star in the direction toward the observer in the continuum and a rotationally broadened spectral-line profile that can be used to derive the radial velocity of the star.

Since the observed radial-velocity curves for OB stars have been derived primarily from hydrogen Balmer absorption lines, we synthesized theoretical radial-velocity curves for the $\mathrm{H} \gamma$ line of the optical star. The radial velocity at a given orbital phase was calculated using the mean wavelength at the $1 / 3,1 / 2$, and $2 / 3$ levels of the maximum depth of the integrated absorption-line profile.

In addition, in order to estimate the uncertainties in the modeling, we carried out computations for the same situations using our previous algorithm for synthesizing theoretical radial-velocity curves, used earlier to analyze the radial-velocity curves of OB supergiants in X-ray binaries with neutron stars [7] and in the Cyg X-1 system [4]. This algorithm was proposed by Antokhina and Cherepashchuk [8] in 1994. The main difference from our more modern algorithm [5, 6] is that the local profile of an area element is found, not by constructing a model atmosphere and calculating the intensity of the outgoing radiation in the line and continuum (taking into account reprocessing of the external $\mathrm{X}$-ray radiation), but instead using computed and tabulated Kurucz Balmer absorptionline profiles for various effective temperatures $T_{\text {eff }}$ and gravitational accelerations $g$. In addition, the effect of heating of the stellar atmosphere by X-ray radiation from its companion was taken into account only in a simple way, by adding the outgoing and incident flux units without taking into account radiative transfer in the stellar atmosphere.

In addition to its simplified treatment of the reflection effect, this method for calculating the hydrogen absorption-line profiles is not entirely correct, since the tables of Kurucz [9] present the theoretical line profiles in relative flux units and not intensities. However, since we are using the theoretical line profiles to derive radial velocities, and not for comparisons with observed spectral lines, we consider this approximation to be acceptable. In addition, calculating the theoretical radial-velocity curves using the algorithm described based on the tables of Kurucz requires comparatively little computer time. The use of the more modern algorithm [5, 6], which calculates a model atmosphere for each local area element, requires appreciably more computer time and became possible only relatively recently with the appearance of computers with processing rates of $1 \mathrm{GHz}$ or higher.

One drawback of the new algorithm is the absence of a contribution to the integrated absorption-line profile of the optical star from the local profiles of areas near the limb of the stellar disk (the requirement of 
Table 2. Mass and corresponding effective temperature of the optical star

\begin{tabular}{c|c|c|c|c|c|c}
\hline \multirow{2}{*}{$m_{v}, M_{\odot}$} & \multirow{2}{*}{$T_{\text {eff }}^{*}, \mathrm{~K}$} & \multirow{2}{*}{$T_{\text {eff }}^{* *}, \mathrm{~K}$} & \multirow{2}{*}{$T_{\text {eff }}^{* * *}, \mathrm{~K}$} & \multicolumn{2}{|c|}{$\begin{array}{c}\text { Range of atmospheric parameter values } \\
\text { on the stellar surface in the Roche model }\end{array}$} & \multirow{2}{*}{$S, \%$} \\
\cline { 4 - 6 } & & & & $\log g$ & $T_{\text {eff }}, \mathrm{K}$ & \\
\hline \multirow{2}{*}{1} & - & 5500 & 5500 & $2.02-3.15$ & $2800-5930$ & 89 \\
5 & 14000 & 12000 & 12000 & $2.02-3.16$ & $6570-12820$ & 93 \\
10 & 17000 & 15000 & 16000 & $2.13-3.22$ & $9190-17060$ & 94 \\
20 & 26000 & 23000 & 23000 & $2.23-3.25$ & $14020-24540$ & 94 \\
30 & 29000 & 28000 & 29000 & $2.28-3.26$ & $18390-31010$ & 96 \\
\hline
\end{tabular}

Note: $T_{\text {eff }}^{*}$ and $T_{\text {eff }}^{* *}$ are the temperatures of the optical star according to the mass-luminosity relations of [11] and [12]; $T_{\mathrm{eff}}^{* * *}$ is the temperature of the optical star used to compute the radial-velocity curves in the current study; and $S$ the fraction of the area of the stellar surface where the temperature differs from the mean effective temperature $T_{\text {eff }}^{* * *}$ by no more than $10 \%$.

the boundary conditions $[5,6])$. At the same time, for real optical stars, the local profiles of areas near the disk limb are nonzero and will make a significant contribution to an integrated absorption-line profile. Note that the old algorithm was free of this effect of "zeroing" the contributions of the local profiles for areas near the disk limb.

We used both these algorithms to synthesize the theoretical radial-velocity curves for all the model problems considered. The results of these computations using these two methods are compared below.

Table 1 presents the parameters of the X-ray binary for which the modeling was carried out. The numerical values of the parameters of the modeled close binary were adopted based on the catalog [10], as being the most characteristic values.

We synthesized radial-velocity curves for optical stars with masses $m_{v}=1,5,10,20,30 M_{\odot}$ (the remaining parameters of the modeled binary system are presented in Table 1). In order to investigate the dependence of the shape of the radial-velocity curve on the orbital inclination $i$, we synthesized radialvelocity curves for $i=30^{\circ}, 60^{\circ}$, and $90^{\circ}$.

When modeling the radial-velocity curves of an optical star with mass $m_{v}=30 M_{\odot}$ using the tables of Kurucz [9], the local gravitational acceleration $g_{\text {loc }}$ and local temperature $T_{\mathrm{loc}}$ at the surface of the optical star fell outside the range of tabulated values, so that there were no tabulated profiles for some of the local areas. The number of such areas was modest (about 10-20\% of the total) and they were all located on the "nose" of the filled Roche lobe of the optical component. In this case, the profiles of all the areas were taken to be the same. We used the profile of the $\mathrm{H} \gamma$ line for the mean effective temperature and the mean gravitational acceleration at the stellar surface as the local profile. The accuracy of this approximation was tested in test computation 1 presented below.

We should also qualify the situation with regard to the mean effective temperature of the optical star. The mean temperature of the Roche lobe filling star is given by the expression $T_{\text {eff }}=\int T_{\text {loc }} d S / \int d S$, where the integration is carried out over the entire surface of the tidally deformed star. Table 2 presents estimates of the effective temperature based on the massluminosity relations of Herrero [11] and Straizhis [12]. We can see that the effective temperatures of the star obtained in different ways are appreciably different. Therefore, we carried out a test computation (test computation 2 ) in order to quantitatively estimate the influence of the effective temperature of the optical star on the theoretical radial-velocity curve. We took the mean effective temperature of the tidally deformed star to be the mean effective temperature of a spherical star with the same volume. The results of the test computations are presented below.

Table 2 also presents the range of effective temperatures and gravitational accelerations at the surface of the optical star in the Roche model. Note that the number of local area elements whose temperature $T_{\text {loc }}$ differs from the adopted effective temperature $T_{\text {eff }}^{* * *}$ by more than $10 \%$ is very small.

\section{Test Computation 1: Influence of the Local-Profile Approximation on the Theoretical Radial-Velocity Curve}

As we indicated above, the test computations check the difference in the radial velocities calculated using the two methods and the tables of Kurucz [9]. We synthesized the radial-velocity curve for an optical 

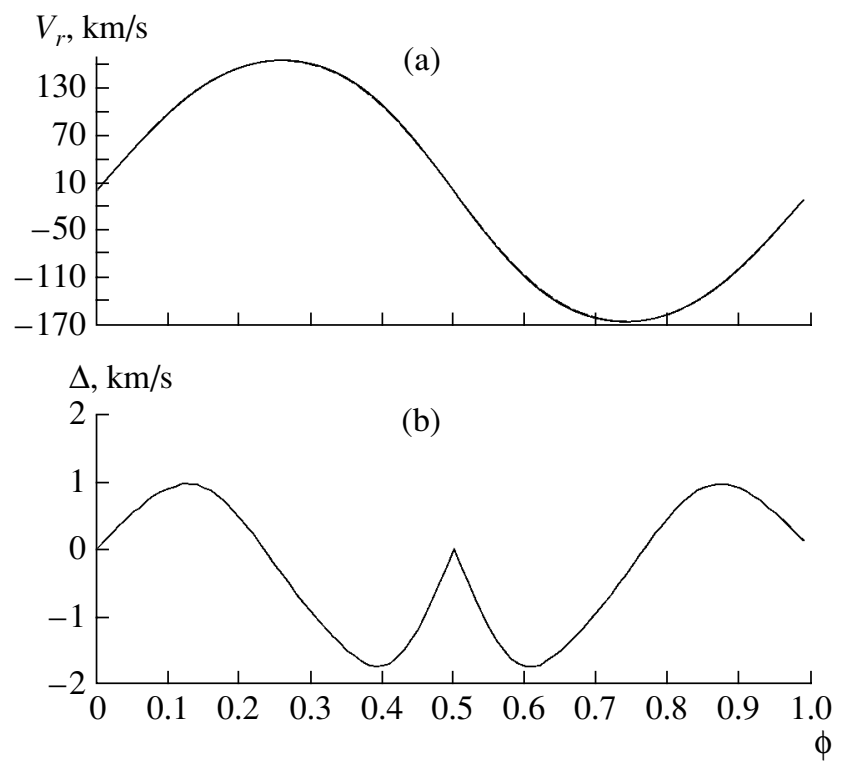

Fig. 1. Test computation 1. (a) Model radial-velocity curve for an optical star with $m_{v}=10 M_{\odot}$ and $T_{\text {eff }}=$ $15000 \mathrm{~K}$ for $i=90^{\circ}$ (solid) together with the same curve obtained assuming that the shape of the local profile is constant (dashed). The two radial-velocity curves virtually coincide on the scale shown. These curves were calculated using the tabulated $\mathrm{H} \gamma$ line profiles (in flux units) of Kurucz. (b) Difference between the absolute values of the radial velocities. See text for more detail.

star with mass $m_{v}=10 M_{\odot}$ and mean effective surface temperature $T_{\text {eff }}=15000 \mathrm{~K}$ (the remaining binary parameters can be found in Table 1); each local area is specified in the LTE approximation in accordance with its local $\mathrm{H} \gamma$ absorption profile from the tables of Kurucz ( solid curve in Fig. 1a). We then constructed the radial-velocity curve for the optical star specifying the shapes of the local profiles for all the area elements to be the same, but taking into account normalization to the continuum over the stellar surface when summing the area profiles. For the constant profile shape, we used the $\mathrm{H} \gamma$ absorption profile for the mean effective temperature and gravitational acceleration of the optical star. In the case of a star with $m_{v}=10 M_{\odot}$, we used the tabulated profile of Kurucz [9] corresponding to $T_{\text {loc }}=15000 \mathrm{~K}$ and $\log g_{\text {loc }}=3.2$. The resulting radial-velocity curve is shown by the dotted curve in Fig. 1a. Figure 1b shows the difference between the absolute values of the radial velocities obtained using the "intrinsic" local profiles and assuming identical local profiles for each area element. We can see that the difference between the curves does not exceed $1.7 \mathrm{~km} / \mathrm{s}$, or $\sim 1 \%$ of the half-amplitude of the radial-velocity curve. An analogous computation was carried out for a close X-ray binary with an optical star with mass
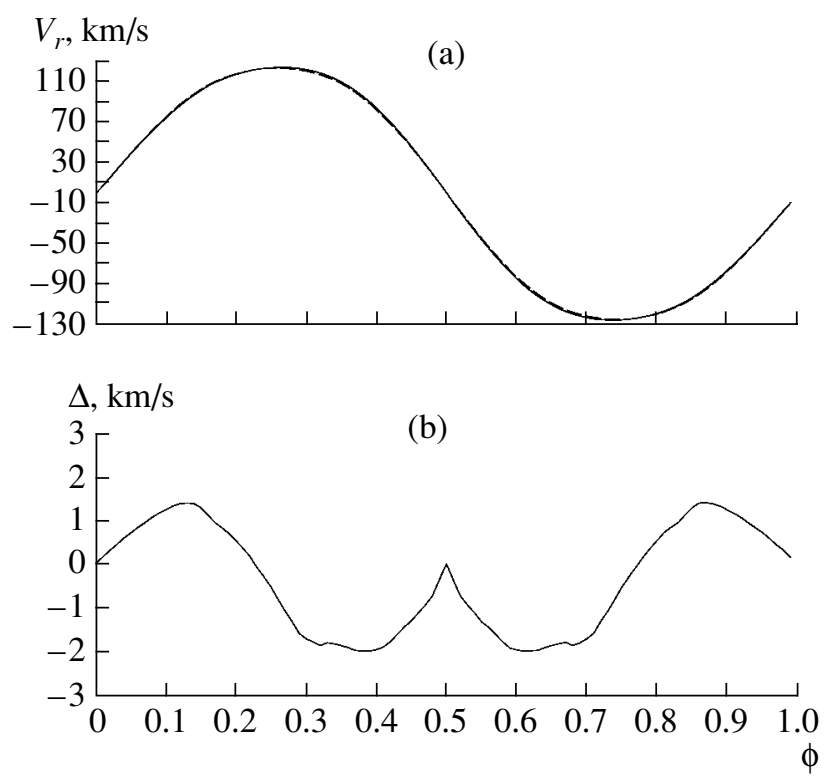

Fig. 2. Same as Fig. 1 for the parameters of the optical star $m_{v}=20 M_{\odot}$ and $T_{\text {eff }}=23000 \mathrm{~K}$.

$m_{v}=20 M_{\odot}$. The resulting radial-velocity curves are shown in Fig. 2a. In this case, the discrepancy between the radial-velocity curves does not exceed $2 \mathrm{~km} / \mathrm{s}$, or $1.6 \%$ of the radial-velocity half-amplitude (Fig. 2b).

Test Computation 2: Influence of the Effective Temperature of the Optical Star on the Theoretical Radial-Velocity Curve

As we noted above, the effective temperature of the optical star is usually not known exactly. We can see from Table 2 that the observed effective temperatures derived from the mass-luminosity relations of $[11$, 12] are somewhat different. Therefore, we carried out a test computation to estimate the influence of the effective temperature of the optical star on the theoretical radial-velocity curve. We synthesized a radial-velocity curve for a close binary with an optical star with mass $m_{v}=10 M_{\odot}$ and orbital inclination $i=90^{\circ}$ for $T_{\text {eff }}=10000 \mathrm{~K}$ and $T_{\text {eff }}=17000 \mathrm{~K}$ (having especially chosen a wide range of variation for the effective temperature). The resulting radial-velocity curves are shown in Fig. 3a (see Table 1 for the remaining binary parameters). Since the discrepancy between the curves is insignificant, Fig. $3 \mathrm{~b}$ presents the difference between their absolute values, $\Delta=$ $\left|V_{r}\left(T_{\text {eff }}=17000 \mathrm{~K}\right)\right|-\left|V_{r}\left(T_{\text {eff }}=10000 \mathrm{~K}\right)\right|$, where $\left|V_{r}\left(T_{\text {eff }}=17000 \mathrm{~K}\right)\right|$ and $\left|V_{r}\left(T_{\text {eff }}=10000 \mathrm{~K}\right)\right|$ are the absolute values of the radial velocity of the optical star 

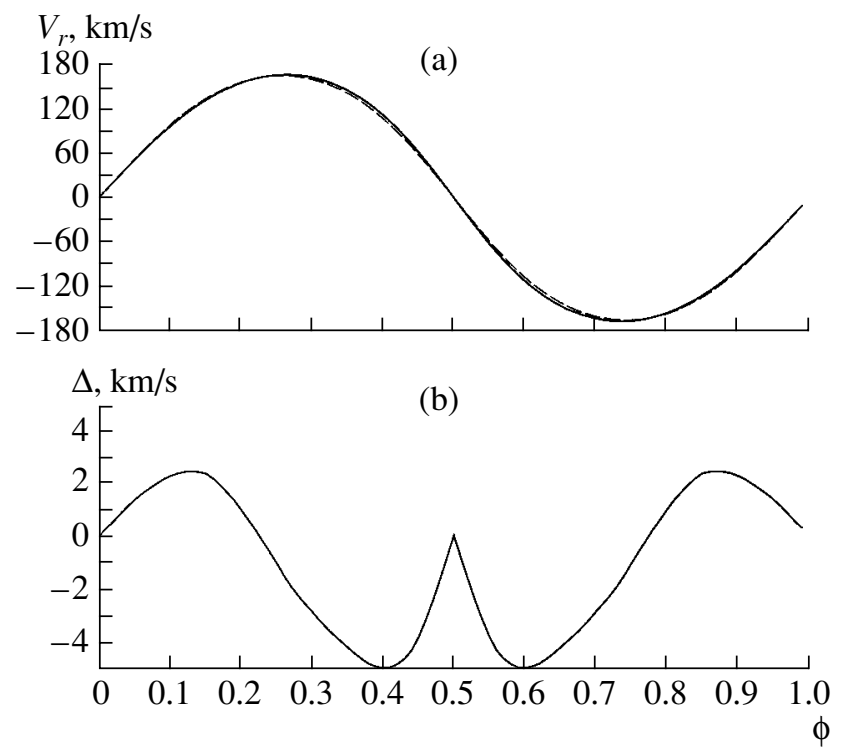

Fig. 3. Test computation 2. (a) Model radial-velocity curves of an optical star with $m_{v}=10 M_{\odot}, i=90^{\circ}$, and $T_{\text {eff }}=10000 \mathrm{~K}$ (solid) and $T_{\text {eff }}=17000 \mathrm{~K}$ (dashed). The curves were calculated using the tabulated $\mathrm{H} \gamma$ profiles (in flux units) of Kurucz. (b) Difference between the absolute values of the radial velocities $\Delta=\mid V_{r}\left(T_{\text {eff }}=\right.$ $17000 \mathrm{~K})|-| V_{r}\left(T_{\text {eff }}=10000 \mathrm{~K}\right) \mid$. See text for more detail.

in the Roche model for mean effective surface temperatures of $T_{\text {eff }}=17000 \mathrm{~K}$ and $T_{\text {eff }}=10000 \mathrm{~K}$. We can see from Fig. $3 \mathrm{~b}$ that the maximum discrepancy between these two values occurs at orbital phases $0.35-0.45$, and reaches $5 \mathrm{~km} / \mathrm{s}$, or $2.6 \%$ of the radialvelocity half-amplitude.

We can see from the test computation that the uncertainty in the mean effective temperature of the optical star ( $\left.\Delta T_{\text {eff }} \simeq 5000-7000 \mathrm{~K}\right)$ has an appreciable effect on the shape of the radial-velocity curve. The variations in the radial-velocity curve are different at different orbital phases (Fig. 3b). These variations are maximum at phases $0.35-0.45$, where they reach $\sim 3 \%$ of the radial-velocity half-amplitude. As we noted above, the uncertainty in the effective temperature was artificially increased in the test computation. According to Table 2, the maximum uncertainty in the effective temperature does not exceed $3000 \mathrm{~K}$, so that the corresponding variations in the radialvelocity curve should not exceed $\sim 1 \%$.

Our computations indicate that the mean effective temperature of the optical star, $T_{\text {eff }}$, should be known as accurately as possible when using radialvelocity curves to determine the parameters of a binary system.

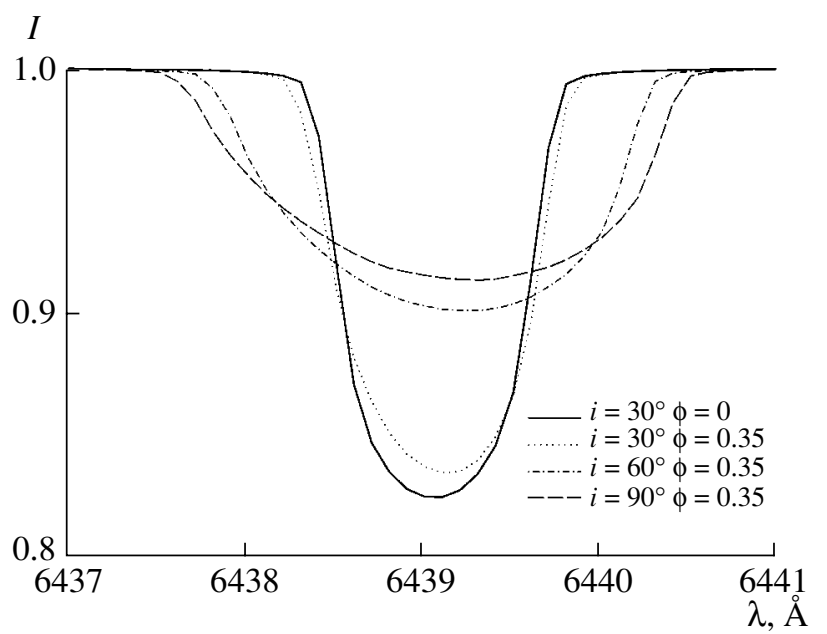

Fig. 4. Theoretical CaI $6439 \AA$ absorption profiles without allowance for an instrumental profile (calculated with the new algorithm $[5,6])$ at orbital phase $\phi=0.0$ for orbital inclination $i=30^{\circ}$ (solid) and at orbital phase $\phi=0.35$ for $i=30^{\circ}$ (dotted), $60^{\circ}$ (dash-dotted), and $90^{\circ}$ (dashed). The model profiles were obtained assuming LTE and a mass and effective temperature for the optical star $m_{v}=1 M_{\odot}$ and $T_{\text {eff }}=5500 \mathrm{~K}$ (see Table 1 for the remaining parameters). The CaI $6439 \AA$ absorption profiles for phase 0.35 have been corrected for the Doppler shifts due to the orbital motion.

\section{DEPENDENCE OF THE SHAPE OF THE RADIAL-VELOCITY CURVE ON THE COMPONENT-MASS RATIO $q$ AND ORBITAL INCLINATION $i$}

We also calculated radial-velocity curves with and without allowing for the influence of the instrumental function on the theoretical integrated profile.

\section{Calculated Radial-Velocity Curves without Account of the Instrumental Function on the Theoretical Absorption Profile}

We synthesized radial-velocity curves in the Roche model assuming LTE for optical stars with masses $m_{v}=1,5,10,20$, and $30 M_{\odot}$ (see Table 1 for the remaining parameters of the $\mathrm{X}$-ray binary). For the optical star with mass $m_{v}=1 M_{\odot}$, the synthesis was carried out for the CaI $6439 \AA$ absorption line. The radial-velocity curve syntheses for the stars with masses $m_{v}=5,10,20$, and $30 M_{\odot}$ were performed for the $\mathrm{H} \gamma$ absorption line using the two methods described above (calculating the intensity of the local profile of each area element based on a constructed model atmosphere $[5,6]$ and based on the tabulated line profiles in flux units of Kurucz [8, 13]). The theoretical integrated $\mathrm{CaI} 6439 \AA$ and $\mathrm{H} \gamma$ absorption profiles are presented in Figs. 4 and 5. For each value of $m_{v}$, we synthesized radial-velocity curves for 


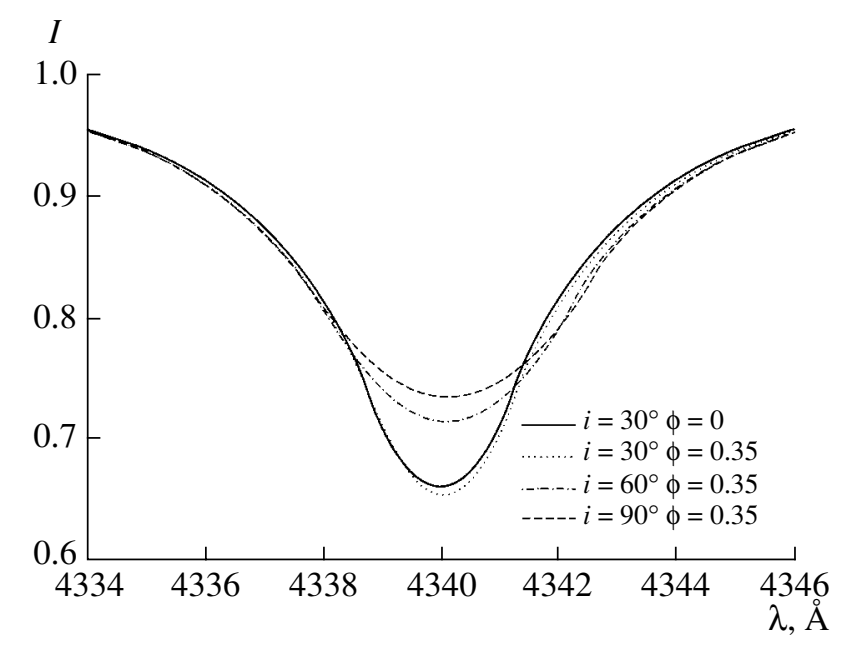

Fig. 5. Same as Fig. 4 for the $\mathrm{H} \gamma$ absorption line with $m_{v}=20 M_{\odot}$ and $T_{\text {eff }}=23000 \mathrm{~K}$.

orbital inclinations $i=30^{\circ}, i=60^{\circ}$, and $i=90^{\circ}$. The resulting theoretical radial-velocity curves for optical stars with masses $m_{v}=1 M_{\odot}, 20 M_{\odot}$, and $30 M_{\odot}$ are presented in Figs. 6a, 7a, and 8a.

The half-amplitude of the radial-velocity curve $K_{v}$ increases with increasing orbital inclination $i$ (Figs. 6a, 7a, 8a). Therefore, each theoretical radialvelocity curve was normalized to its value of $K_{v}$, which was taken to be the maximum radial velocity in the orbital-phase interval $0.0-0.5$. The normalized radial-velocity curves for optical stars with masses $m_{v}=1,20,30 M_{\odot}$ are presented in Figs. 6b, 7b, 8b. Since the effect of the differences in the shapes of the curves is small (relative to the half-amplitude $K_{v}$ ), we calculated the differences of the absolute values of the normalized radial velocities $\Delta=\left|V_{\text {norm }}\left(i=90^{\circ}\right)\right|-\left|V_{\text {norm }}\left(i=30^{\circ}\right)\right|$ and $\Delta=$ $\left|V_{\text {norm }}\left(i=90^{\circ}\right)\right|-\left|V_{\text {norm }}\left(i=60^{\circ}\right)\right|$, where $\mid V_{\text {norm }}(i=$ $\left.90^{\circ}\right)|,| V_{\text {norm }}\left(i=60^{\circ}\right) \mid$, and $\left|V_{\text {norm }}\left(i=30^{\circ}\right)\right|$ are the absolute values of the normalized radial velocities for orbital inclinations of $i=90^{\circ}, i=60^{\circ}$, and $i=30^{\circ}$. The maximum differences between the normalized radial-velocity curves obtained using the two methods for optical stars with masses $m_{v}=1,20$, $30 M_{\odot}$ are reached at orbital phases $0.35-0.45$ and $0.55-0.65$ (Tables 3 and 4 ). We denote $\Delta_{I}$ and $\Delta_{F}$ to be the maximum variations in the shape of the radialvelocity curve for variations in the orbital inclination obtained using the new algorithm of $[5,6]$ and the theoretical line profiles of Kurucz [8, 13], respectively.

The difference in the absolute values of the normalized radial velocities for optical stars with masses $m_{v}=1,20,30 M_{\odot}$ are presented in Figs. $6 \mathrm{c}, 7 \mathrm{c}, 8 \mathrm{c}$. We can see from these figures that the maximum difference between the radial-velocity curves obtained
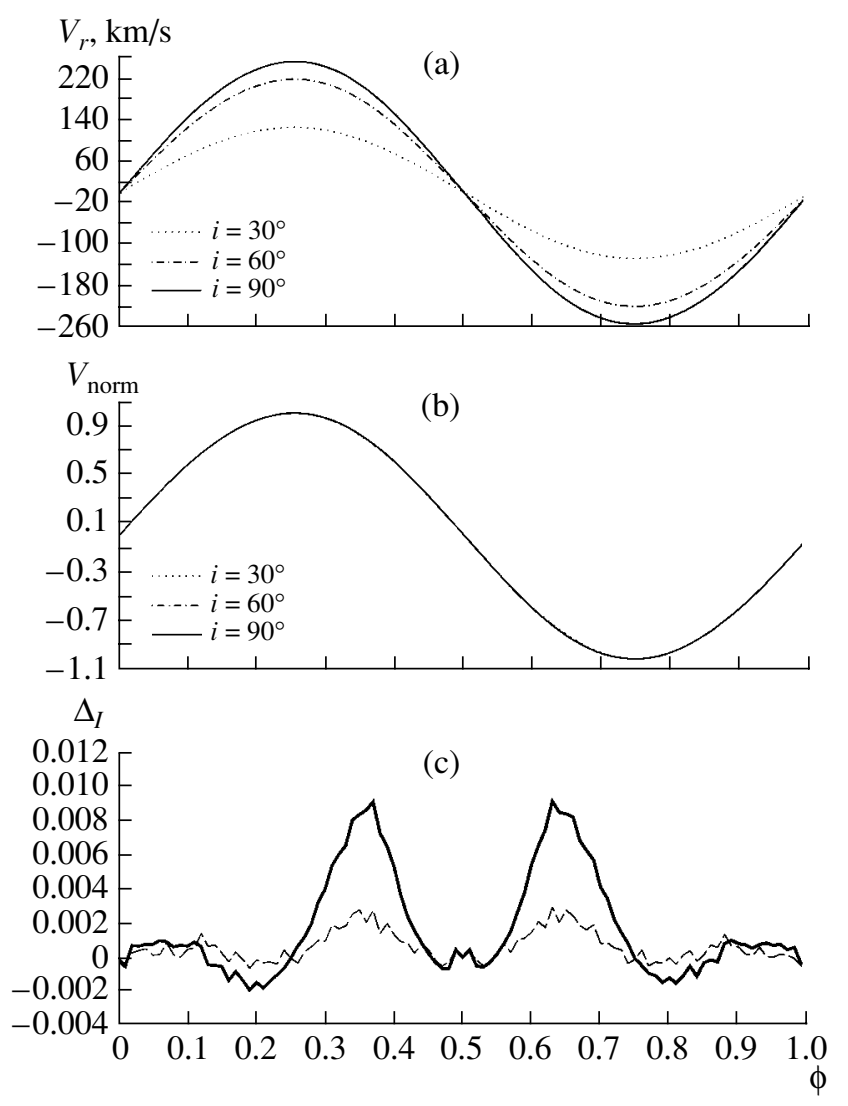

Fig. 6. (a) Theoretical radial-velocity curve of an optical star with $m_{v}=1 M_{\odot}, T_{\text {eff }}=5500 \mathrm{~K}$ calculated without allowance for the effect of the instrumental function on the integrated CaI $6439 \AA$ absorption profile for orbital inclinations $i=30^{\circ}$ (dotted), $60^{\circ}$ (dash-dotted), and $90^{\circ}$ (solid) (see Table 1 for remaining parameters). The radial-velocity curves were calculated using the new algorithm [5, 6]. (b) The same curves normalized to their half-amplitudes for $i=30^{\circ}$ (dotted), $60^{\circ}$ (dashdotted), and $90^{\circ}$ (solid). The curves nearly coincide on this scale. (c) Difference between the absolute values of the normalized radial velocities $\Delta_{I}=\left|V_{\text {norm }}\left(i=90^{\circ}\right)\right|-$ $\left|V_{\text {norm }}\left(i=60^{\circ}\right)\right|$ (dashed) and $\Delta_{I}=\left|V_{\text {norm }}\left(i=90^{\circ}\right)\right|-$ $\left|V_{\text {norm }}\left(i=30^{\circ}\right)\right|$ (solid). The quantity $\Delta_{I}$ is given in units of the half-amplitude of the radial-velocity curve $K_{v}$ (for more detail, see text).

for different values of $i$ occurs at orbital phases $0.35-0.45$ and $0.55-0.65$.

It follows from the computations that the variations in the shape of the radial-velocity curve depend on both the orbital inclination $i$ and the componentmass ratio $q=m_{x} / m_{v}$. For example, the maximum variations in this curve (in units of the radial-velocity half-amplitude) when the inclination is changed from $i=30^{\circ}$ to $i=90^{\circ}$ for optical stars with masses $m_{v}=$ $1,5,10,20$, and $30 M_{\odot}$ are $\Delta_{F}=0.9,3.2,4.6,5.7$, and $8 \%$, respectively.

Thus, the variations in the shape of the radial- 

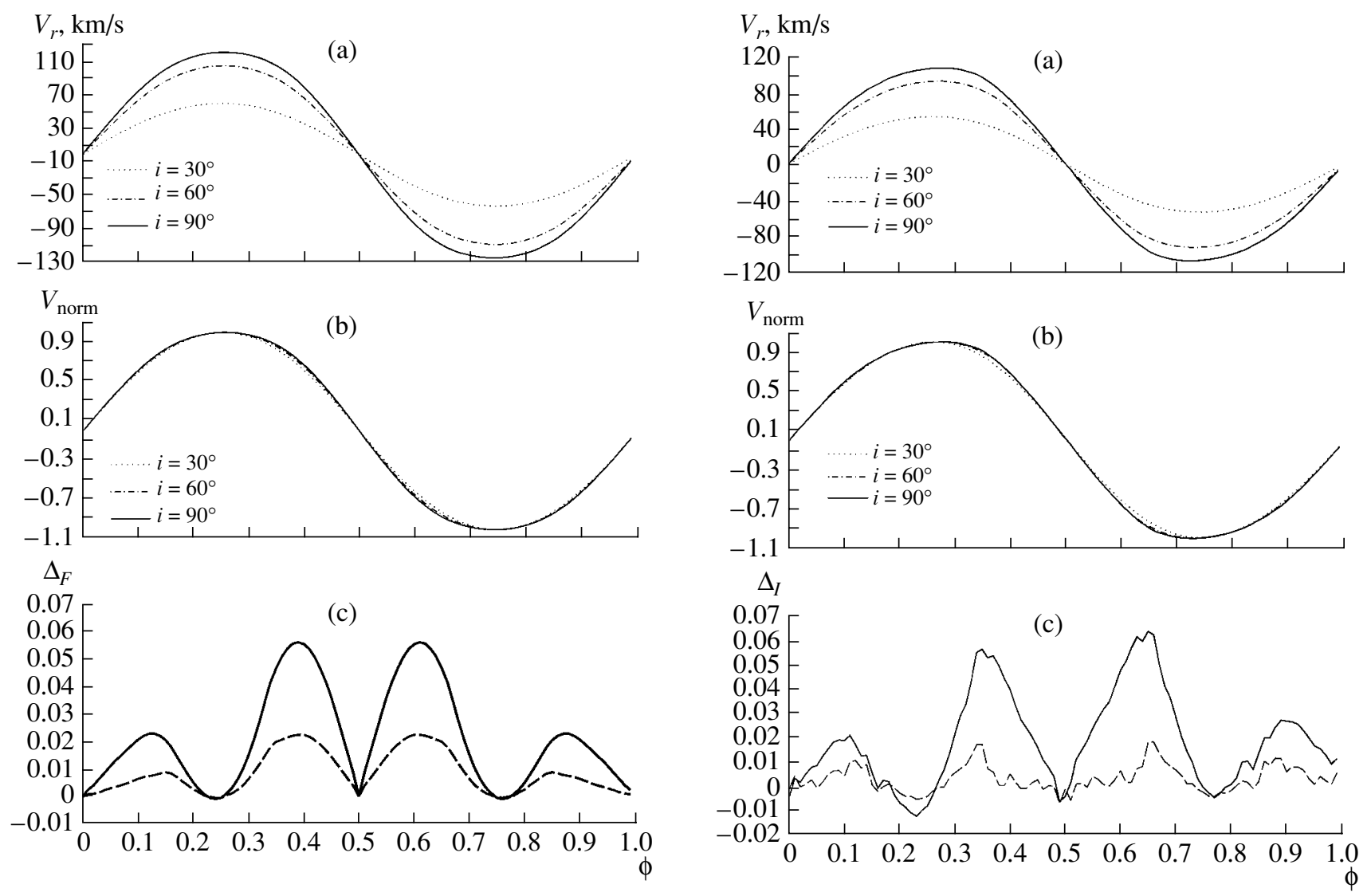

Fig. 7. (a) Theoretical radial-velocity curve of an optical star with $m_{v}=20 M_{\odot}, T_{\text {eff }}=23000 \mathrm{~K}$ calculated without allowance for the effect of the instrumental function on the integrated $\mathrm{H} \gamma$ absorption profile for orbital inclinations $i=30^{\circ}$ (dotted), 60 $0^{\circ}$ (dash-dotted), and $90^{\circ}$ (solid) (see Table 1 for remaining parameters). The radial-velocity curves were calculated using the old algorithm [8, 13] (using the tabulated $\mathrm{H} \gamma$ absorption profiles of Kurucz in flux units). (b) The same curves normalized to their half-amplitudes for $i=30^{\circ}$ (dotted), 60 ${ }^{\circ}$ (dash-dotted), and $90^{\circ}$ (solid). (c) Difference between the absolute values of the normalized radial velocities $\Delta_{F}=\left|V_{\text {norm }}\left(i=90^{\circ}\right)\right|-\left|V_{\text {norm }}\left(i=60^{\circ}\right)\right|$ (dashed) and $\Delta_{F}=\left|V_{\text {norm }}\left(i=90^{\circ}\right)\right|-\left|V_{\text {norm }}\left(i=30^{\circ}\right)\right|$ (solid). The quantity $\Delta_{F}$ is given in units of the halfamplitude of the radial-velocity curve $K_{v}$ (for more detail see text).

velocity curve with changes in the orbital inclination are most clearly expressed in systems with low component-mass ratios $q=m_{x} / m_{v}$. This is due to the fact that, when $q<1$, the center of mass of the binary system lies inside the body of the optical star, so that, during the orbital motion, the part of the stellar surface lying inside the inner Lagrangian point moves in the same direction as the relativistic object. This leads to strong distortion of the integrated absorption-line profile that depends on both $i$ and $q$.

Fig. 8. Same as Fig. 6 for the $\mathrm{H} \gamma$ absorption line and with $m_{v}=30 M_{\odot}$ and $T_{\text {eff }}=29000 \mathrm{~K}$.

\section{Computation of Radial-Velocity Curves Including the Effect of the Instrumental Function on the Theoretical Absorption Profiles}

As in the previous case, the radial-velocity curve synthesis was carried out for optical stars with masses $m_{v}=1,5,10,20$, and $30 M_{\odot}$ (see Table 1 for the remaining binary parameters). We used a Gaussian profile for the instrumental function of the spectrograph. The CaI $6439 \AA$ line was convolved with an instrumental function with a full width at half maximum intensity $\mathrm{FWHM}=1 \AA$. When synthesizing the curves for stars with masses $m_{v}=$ $5,10,20$, and $30 M_{\odot}$, the theoretical $\mathrm{H} \gamma$ profile was convolved with an instrumental function with $F W H M=7 \AA$. The convolved theoretical CaI $6439 \AA$ and $\mathrm{H} \gamma$ absorption profiles are presented in Figs. 9 and 10 , respectively.

We investigated the variations of the radialvelocity curves with variations of the orbital inclination analogous to those described above. As earlier, the maximum changes in the curves occurred at phases $0.35-0.45$. The variations in the radialvelocity curve obtained when the inclination was changed from $i=60^{\circ}$ to $i=90^{\circ}$ are given in Table 5 . 
Table 3. Maximum variation of the shape of the radialvelocity curve in the Roche model for variations of the orbital inclination from $60^{\circ}$ to $90^{\circ}$

\begin{tabular}{c|c|c}
\hline$m_{v}, M_{\odot}$ & $\Delta_{I}, \%$ & $\Delta_{F}, \%$ \\
\hline 1 & 0.3 & - \\
5 & 0.4 & 1.1 \\
10 & 0.6 & 1.5 \\
20 & 1.1 & 2.3 \\
30 & 1.9 & 2.9 \\
\hline
\end{tabular}

Note: The quantities $\Delta_{I}$ and $\Delta_{F}$ are expressed in units of the half-amplitude $K_{v}$. See text for more detail.

The analogous results for the case when the inclination is changed from $i=30^{\circ}$ to $i=90^{\circ}$ are given in Table 6. A comparison of Tables 3, 4 and 5, 6 shows that the results obtained with and without the effect of the instrumental profile coincide within reasonable errors for the computed integrated profiles.

Thus, the variations in the shape of the radialvelocity curve with orbital inclination are not "washed out" by the instrumental function of the spectrograph, leaving intact the possibility of estimating the orbital inclination of a binary system using accurate observations of the radial-velocity curve. Estimating the orbital inclination of a system with a given mass for its optical star reduces to a required accuracy for the radial-velocity observations. For example, for binary systems with optical-star masses $m_{v}=20-30 M_{\odot}$ (close to filling their Roche lobes), the accuracy in the radial velocities must be better than $7-8 \%$ of the halfamplitude of the radial-velocity curve, $K_{v}$. For sys-

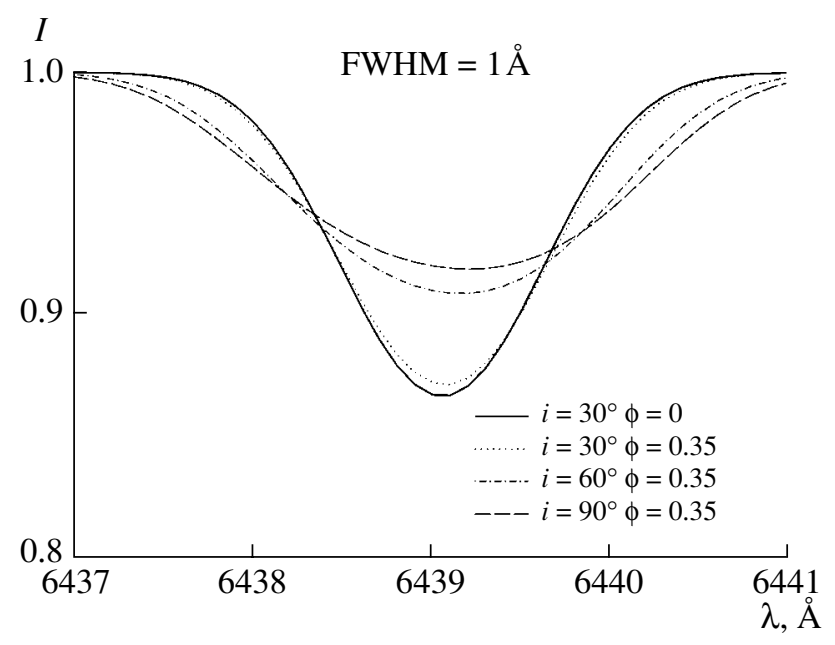

Fig. 9. Same as Fig. 4 but including the effect of an instrumental profile with FWHM $=1 \AA$.
Table 4. Maximum variation of the shape of the radialvelocity curve in the Roche model for variations of the orbital inclination from $30^{\circ}$ to $90^{\circ}$

\begin{tabular}{c|c|c}
\hline$m_{v}, M_{\odot}$ & $\Delta_{I}, \%$ & $\Delta_{F}, \%$ \\
\hline 1 & 0.9 & - \\
5 & 1.5 & 3.2 \\
10 & 2.5 & 4.6 \\
20 & 4.2 & 5.7 \\
30 & 6.5 & 8.0 \\
\hline
\end{tabular}

Note: The quantities $\Delta_{I}$ and $\Delta_{F}$ are expressed in units of the half-amplitude $K_{v}$. See text for more detail.

tems with optical-star masses $m_{v}=1-10 M_{\odot}$ (close to filling their Roche lobes), this accuracy must be better than $1-4 \%$ of $K_{v}$. The orbital inclination of the Cyg X-1 system estimated using an accurate radial-velocity curve is presented in [4]; the accuracy in the observed radial velocities $\sigma_{V_{r}}$ was $\sim 3 \%$ of the half-amplitude $K_{v}$. For an optical-star mass of $m_{v} \simeq 20 M_{\odot}$, this made it possible to place a limit on the orbital inclination of $i<45^{\circ}$ based purely on the observed radial-velocity curve.

\section{FITTING THE OBSERVED RADIAL-VELOCITY CURVE OF THE Cyg X-1 SYSTEM}

The fitting of the mean radial-velocity curve for Cyg X-1 presented in [4] was carried out with the earlier algorithm, which used the tabulated $\mathrm{H} \gamma$ profiles of Kurucz [9] in fluxes for the local profiles of the area elements. However, as we can see from Tables 3-6, the effects of varying the shapes of the radial-velocity curves calculated using the old and new algorithms are somewhat different. For this reason, we decided to fit the accurate mean radial-velocity curve considered in [4] using the new algorithm, in which the integrated $\mathrm{H} \gamma$ absorption profiles are calculated in intensity units based on model atmospheres for the local area elements $[5,6]$. The calculations based on the old algorithm yielded a mass for the compact object in the Cyg X-1 binary of $m_{x}=9.0-13.2 M_{\odot}$ [4]. No model for the binary system with an orbital inclination $i<45^{\circ}$ is consistent with the accurate observational radial-velocity curve [4].

We used the Fisher statistical criterion to test the adequacy of the model description of the observational data. The method used is described in detail in [4], and we do not present it here. As in our earlier work [4], the test of the model description of the observational data was made for the $\alpha=5 \%$ significance level. 
Table 5. Maximum variation of the shape of the radialvelocity curve in the Roche model for variations of the orbital inclination from $60^{\circ}$ to $90^{\circ}$, including the effect of the instrumental function on the theoretical integrated absorption profile

\begin{tabular}{c|c|c}
\hline$m_{v}, M_{\odot}$ & $\Delta_{I}, \%$ & $\Delta_{F}, \%$ \\
\hline 1 & 0.3 & - \\
5 & 0.4 & 1.1 \\
10 & 0.6 & 1.4 \\
20 & 1.1 & 1.7 \\
30 & 2.0 & 3.0 \\
\hline
\end{tabular}

Note: The quantities $\Delta_{I}$ and $\Delta_{F}$ are expressed in units of the half-amplitude $K_{v}$. See text for more detail.

Fits of the radial-velocity curve using the new algorithm were obtained both with and without including the effect of the instrumental function on the model integrated $\mathrm{H} \gamma$ absorption profile of the optical star. The width of the instrumental function was taken to be FWHM $=7 \AA$. Fitting was carried out for masses of the optical star $m_{v}=20,30,40,50$, and $60 M_{\odot}$; the remaining model parameters are given in Table 2 in [4]. This analysis yielded dependences between the masses of the optical star and of the relativistic object for orbital inclinations $i=30^{\circ}, 35^{\circ}$, $40^{\circ}, 45^{\circ}, 55^{\circ}$, and $65^{\circ}$, presented in Fig. 11.

As before, the results for orbital inclinations $i<45^{\circ}$ are rejected at the $5 \%$ significance level. The mass estimates obtained are presented in Tables 7 and 8.

If the mass of the optical star is $m_{v}=$ $22 M_{\odot}[4,11]$, the orbital inclination does not exceed $i=43^{\circ}$ (Fig. 11). Since analysis of the photometric light curve places a lower limit on the orbital inclination of $i>31^{\circ}$ [14], we can conclude that the mass of the relativistic object in the Cyg X-1 binary (for a mass of the optical star $m_{v}=22 M_{\odot}$ ) lies in the ranges $m_{x}=8.4-12.8 M_{\odot}$ (Fig. 11a) and $m_{x}=8.2-12.6 M_{\odot}$ (Fig. 11b). Recall that our earlier results for Cyg X-1 [4] indicated an orbital inclination $i<45^{\circ}$ and a mass of the relativistic object $m_{x}=9.0-13.2 M_{\odot}$ (for a mass of the optical star $\left.m_{v}=22 M_{\odot}\right)$. Thus, the results obtained for the new (more correct) $[5,6]$ and old [8, 13] algorithms are in good agreement.

Based on the example of the Cyg X-1 system, we can see that the accuracy of the mean observed radialvelocity curve, $3 \%$ ( of a half-amplitude of $\sim 75 \mathrm{~km} / \mathrm{s}$ ), was sufficient to place constraints on the orbital inclination of the binary system and estimate the mass of the black hole based on a single radial-velocity curve.
Table 6. Maximum variation of the shape of the radialvelocity curve in the Roche model for variations of the orbital inclination from $30^{\circ}$ to $90^{\circ}$, including the effect of the instrumental function on the theoretical integrated absorption profile

\begin{tabular}{c|c|c}
\hline$m_{v}, M_{\odot}$ & $\Delta_{I}, \%$ & $\Delta_{F}, \%$ \\
\hline 1 & 0.9 & - \\
5 & 1.6 & 3.3 \\
10 & 2.4 & 4.2 \\
20 & 4.2 & 5.5 \\
30 & 6.8 & 8.7 \\
\hline
\end{tabular}

Note: The quantities $\Delta_{I}$ and $\Delta_{F}$ are expressed in units of the half-amplitude $K_{v}$. See text for more detail.

\section{DISCUSSION}

The model computations show that the variations in the shape of the radial-velocity curve of the optical star with variations in the orbital inclination are more clearly expressed in binaries with massive optical components (or low component-mass ratios $q=$ $m_{x} / m_{v}$ ). In addition, these variations are qualitatively similar for optical stars with low, moderate, and high masses - the largest variations (in units of the halfamplitude $K_{v}$ ) occur at orbital phases $0.35-0.45$ and $0.55-0.65$.

The variations in the shape of the radial-velocity curve are related to variations in the absorption-line profiles used to determine the radial velocities. Recall that the radial velocities were determined for the mean wavelength found for three levels: $1 / 3,1 / 2$, and $2 / 3$ of the maximum depth of the absorption line. The

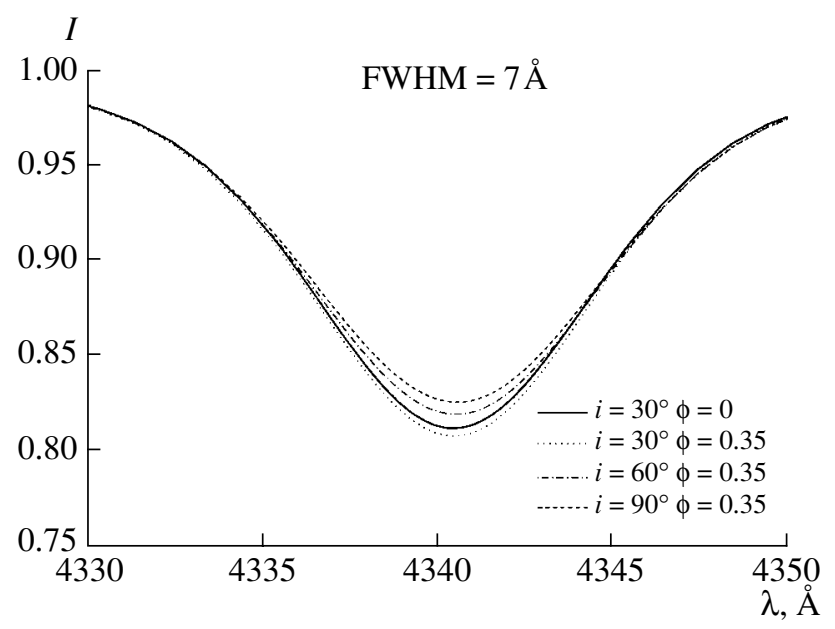

Fig. 10. Same as Fig. 5 but including the effect of an instrumental profile with FWHM $=7 \AA$. 

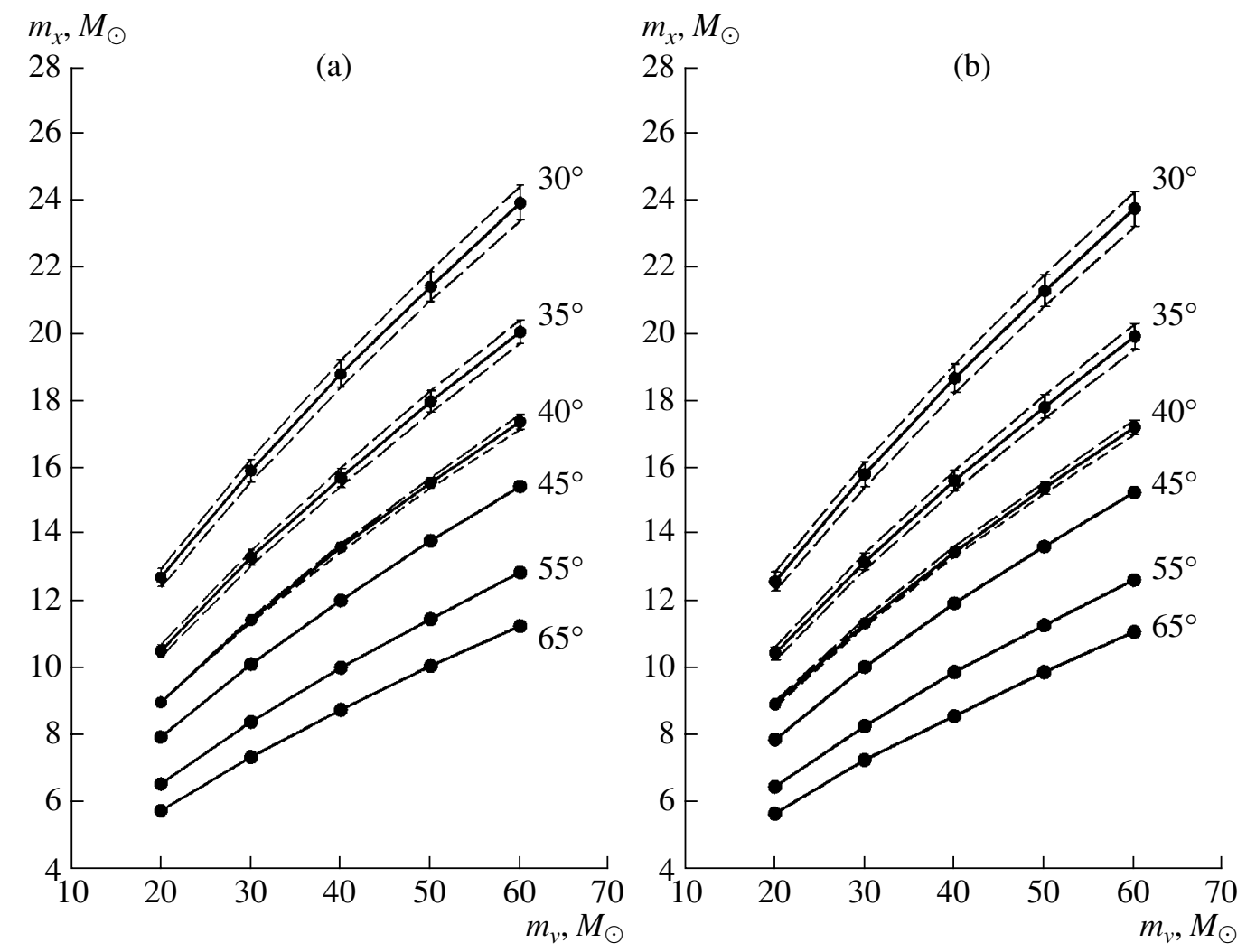

Fig. 11. (a) Dependence of the mass of the compact object in the X-ray binary Cyg X-1 on the mass of the optical star for orbital inclinations $i=30^{\circ}, 35^{\circ}, 40^{\circ}, 45^{\circ}, 55^{\circ}$, and $65^{\circ}$, obtained in the Roche model fitting the mean high-accuracy radial-velocity curve from [4]. The orbital inclination is indicated next to the corresponding curve. (b) Same as (a) but including the effect of an instrumental profile with FWHM $=7 \AA$ on the calculated integrated $\mathrm{H} \gamma$ profile of the optical star. The new algorithm of [5, 6] was used when calculating the integrated $\mathrm{H} \gamma$ profiles and radial-velocity curves.

shape of an absorption line associated with the optical star in a close binary undergoes variations due to both its orbital motion and changes in the line of sight from the observer toward the system. The dependence of the shape of such an absorption profile due to the star's orbital motion is discussed in [1-3] and is associated with variations in the shape of the projection of the optical star onto the plane of the sky. Let us now consider how variations in the binary orbital inclination $i$ affect the shape of the absorptionline profiles and radial-velocity curve.

An optical star in a binary that is close to filling its Roche lobe is tidally deformed and has complex distributions of the velocity, temperature, and gravitational acceleration over its surface. Therefore, regions of the optical component with qualitatively different parameters are projected onto the plane of the sky of the observer when the orbital inclination $i$ is varied (Fig. 12). This leads to variations in the absorption profiles, which, in turn, lead to variations in the radialvelocity curve. Figures 4 and 5 present the integrated absorption profiles for the CaI $6439 \AA$ and $\mathrm{H} \gamma$ lines obtained without including the effect of the instrumental profile for orbital inclinations $i=30^{\circ}, 60^{\circ}$, and $90^{\circ}$. It is clear that the profiles obtained for the different orbital inclinations are different. The profiles are appreciably asymmetric, and this asymmetry increases with increasing values of $i$.

The dependence of the absorption profiles and radial-velocity curve on $i$ grows as the componentmass ratio $q$ decreases. Returning to the computational results, we see that, when $q=1-10$, the variations in the radial-velocity curve are $\sim 1-4 \%$ (Fig. 6), while, when $q=0.3-0.5$, these variations increase to $\sim 4-8 \%$ (Figs. 7 and 8 ). It is clear that the magnitude of the dependence of the radial-velocity curve on $i$ grows with decreasing $q=m_{x} / m_{v}$. This is due to the spatial disposition of the center of mass of the binary relative to the body of the optical star. As $q$ decreases, the barycenter of the binary system shifts toward the optical component, with the center of mass lying inside the body of the optical star when $q<1$. In this case $(q<1)$, the nose of the optical star moves in the same direction along the orbit as the 
Table 7. Mass of the relativistic component as a function of the mass of the optical star for the Roche model and orbital inclinations $i=30^{\circ}, 35^{\circ}, 40^{\circ}, 45^{\circ}, 55^{\circ}$, and $65^{\circ}$ based on the model integrated $\mathrm{H} \gamma$ profiles, without including the instrumental function

\begin{tabular}{c|c|c|c|c|c|c}
\hline \multirow{2}{*}{$m_{v}, M_{\odot}$} & \multicolumn{7}{c}{$m_{x}, M_{\odot}$} \\
\cline { 2 - 6 } & $i=30^{\circ}$ & $i=35^{\circ}$ & $i=40^{\circ}$ & $i=45^{\circ}$ & $i=55^{\circ}$ & $i=65^{\circ}$ \\
\hline \multirow{2}{*}{20} & $12.75_{-0.30}^{+0.25}$ & $10.55_{-0.17}^{+0.19}$ & $9.03_{-0.02}^{+0.02}$ & 8.0 & 6.60 & 5.80 \\
30 & $15.97_{-0.32}^{+0.36}$ & $13.35_{-0.25}^{+0.20}$ & $11.47_{-0.07}^{+0.08}$ & 10.15 & 8.45 & 7.40 \\
40 & $18.85_{-0.40}^{+0.40}$ & $15.75_{-0.25}^{+0.30}$ & $13.65_{-0.15}^{+0.10}$ & 12.05 & 10.05 & 8.80 \\
50 & $21.45_{-0.40}^{+0.50}$ & $18.03_{-0.33}^{+0.32}$ & $15.60_{-0.15}^{+0.15}$ & 13.85 & 11.50 & 10.10 \\
60 & $23.95_{-0.55}^{+0.50}$ & $20.10_{-0.35}^{+0.35}$ & $17.43_{-0.23}^{+0.22}$ & 15.50 & 12.90 & 11.30 \\
\hline
\end{tabular}

Note: The confidence intervals for $m_{x}$ for $i=45^{\circ}, 55^{\circ}$, and $65^{\circ}$ are not indicated, since the model of the binary system is rejected at the $\alpha=5 \%$ significance level in these cases.

Table 8. Mass of the relativistic component as a function of the mass of the optical star for the Roche model and orbital inclinations $i=30^{\circ}, 35^{\circ}, 40^{\circ}, 45^{\circ}, 55^{\circ}$, and $65^{\circ}$ based on the model integrated $\mathrm{H} \gamma$ profiles, including an instrumental function with $\mathrm{FWHM}=7 \AA$

\begin{tabular}{c|c|c|c|c|c|c}
\hline \multirow{2}{*}{$m_{v}, M_{\odot}$} & \multicolumn{7}{|c}{$m_{x}, M_{\odot}$} \\
\cline { 2 - 7 } & $i=30^{\circ}$ & $i=35^{\circ}$ & $i=40^{\circ}$ & $i=45^{\circ}$ & $i=55^{\circ}$ & $i=65^{\circ}$ \\
\hline \multirow{2}{*}{20} & $12.60_{-0.28}^{+0.30}$ & $10.45_{-0.20}^{+0.20}$ & $8.95_{-0.10}^{+0.10}$ & 7.9 & 6.50 & 5.70 \\
30 & $15.83_{-0.38}^{+0.37}$ & $13.18_{-0.23}^{+0.27}$ & $11.35_{-0.10}^{+0.15}$ & 10.05 & 8.30 & 7.30 \\
40 & $18.70_{-0.45}^{+0.40}$ & $15.65_{-0.30}^{+0.30}$ & $13.48_{-0.13}^{+0.17}$ & 11.95 & 9.90 & 8.60 \\
50 & $21.30_{-0.45}^{+0.50}$ & $17.85_{-0.35}^{+0.35}$ & $15.43_{-0.18}^{+0.17}$ & 13.65 & 11.30 & 9.90 \\
60 & $23.75_{-0.55}^{+0.50}$ & $19.95_{-0.40}^{+0.35}$ & $17.25_{-0.22}^{+0.23}$ & 15.30 & 12.65 & 11.10 \\
\hline
\end{tabular}

Note: The confidence intervals for $m_{x}$ for $i=45^{\circ}, 55^{\circ}$, and $65^{\circ}$ are not indicated, since the model of the binary system is rejected at the $\alpha=5 \%$ significance level in these cases.

relativistic object, leading to the maximum distortion of the total absorption profiles of the optical star. As a consequence, the shape of the light curve is more sensitive to variations in the orbital inclination when $q<1$ than when $q>1$.

The size of the projection of the optical star onto the plane of the sky varies during its orbital motion. The degree of asymmetry of the absorption profiles depends not only on the asymmetry of the velocity field of the area elements and the distributions of $T_{\text {loc }}$ and $\log g_{\text {loc }}$ relative to the geometrical center of the projection, but also on gradients of these parameters over the surface of the optical component. The most extreme region from the point of view of its physical parameters and their gradients is the nose of the optical star (which is close to filling its Roche lobe). At orbital phases $0.35-0.45$ and $0.55-0.65$, a large fraction of this nose is visible to the observer. Therefore, the distributions of $T_{\text {loc }}$ and $\log g_{\text {loc }}$ and their gradients are maximally asymmetric relative to the geometrical center of the projected star. This makes the integrated absorption profiles of the optical star maximally asymmetric, and hence maximally sensitive to variations in the orbital inclination, at orbital phases $0.35-0.45$ and $0.55-0.65$ (Figs. 4 and 5).

Thus, the strongest orbital variability in the absorption profiles in the spectrum of the optical star is observed at orbital inclinations $i \simeq 90^{\circ}$ and for component-mass ratios $q<1$, since, in this case, the nose of the optical star is eclipsed by the star's body 

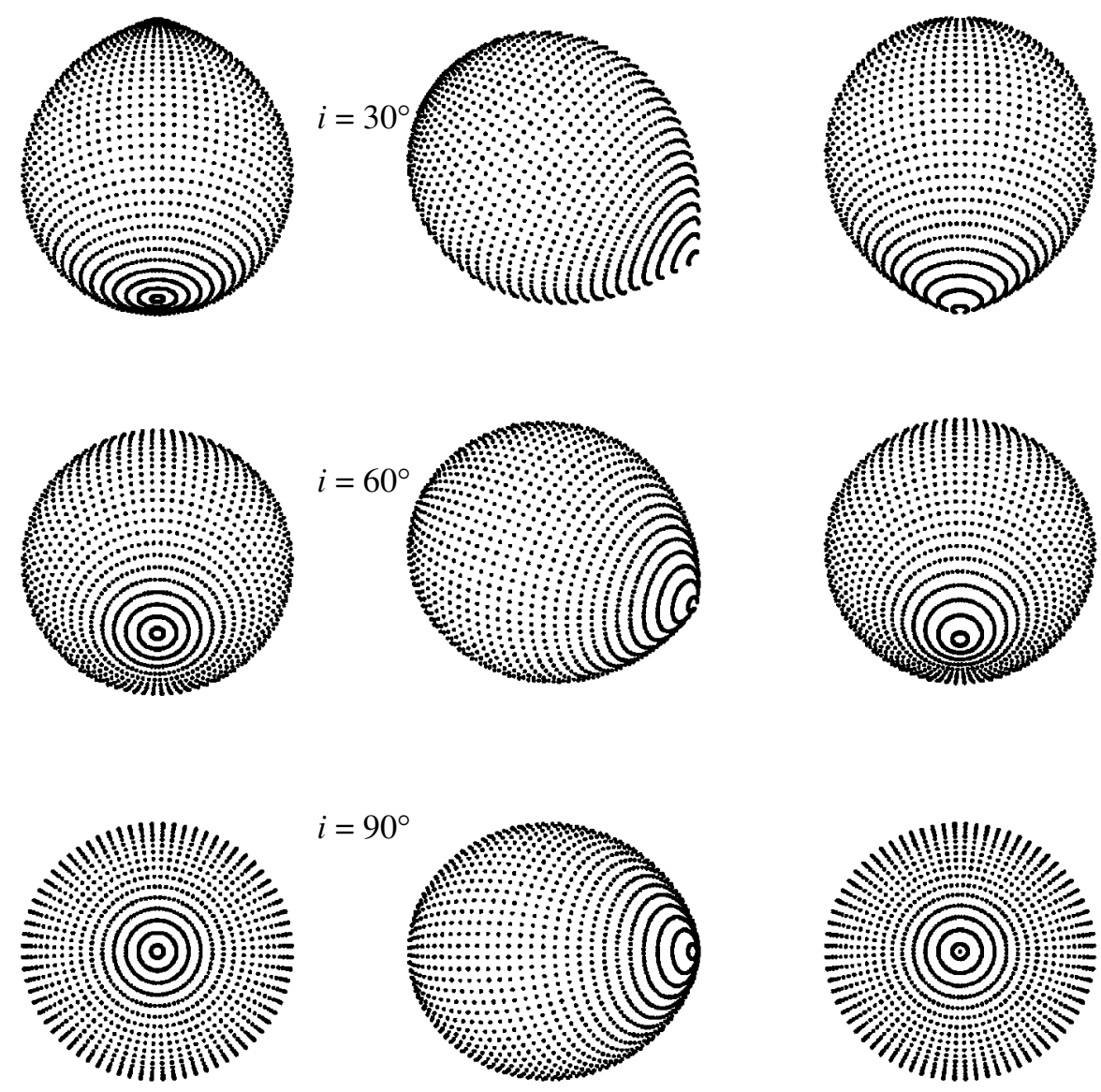

$$
\phi=0
$$

$\phi=0.35$

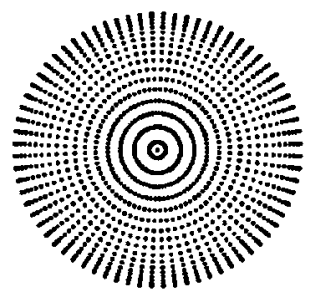

$\phi=0.50$

Fig. 12. Optical star in an X-ray binary in a Roche model with $q=1$, a degree of filling of the Roche lobe $\mu=1$, and orbital inclinations $i=30^{\circ}, 60^{\circ}, 90^{\circ}$ at orbital phases $0.0,0.35,0.5$. When $i=90^{\circ}$, the projection of the star onto the plane of the sky varies strongly (from being circular to pear-shaped). The "nose" of the star is sometimes eclipsed and sometimes not, leading to strong distortion of the line profiles and radial-velocity curve. When $i=30^{\circ}$, the shape of the projection of the star onto the plane of the sky is nearly constant and varies only with the position angle of the figure, which does not influence the shape of the radial-velocity curve.

during the orbital motion. The shape of the star's projection onto the plane of the sky varies strongly when $i=90^{\circ}$, from being circular to pear-shaped, which gives rise to the maximum asymmetry in the radialvelocity curve. When $i \ll 90^{\circ}$, the nose of the optical star is not eclipsed during the orbital motion. The projections onto the plane of the sky of the shape of the star and of regions on its surface remain nearly constant (only the orientation of the stellar shape varies), making the line profiles less sensitive to the orbital shifts of the optical star. This is illustrated in Fig. 12.

\section{CONCLUSION}

The main result of this study is our qualitative estimate of the variations of the radial-velocity curve of the optical star in a close binary system as a function of the orbital inclination $i$ and component-mass ratio $q$ for stars with various masses and temperatures (Tables 3-6).

Another important result is our new fitting of an accurate radial-velocity curve for the optical star in the Cyg X-1 binary system using a new and more correct algorithm to synthesize the theoretical radialvelocity curve (based on calculating model atmospheres for each area element $[5,6])$. The computations show that the accuracy of the radial-velocity curve in this case $(\sim 3 \%$ of the radial-velocity halfamplitude $K_{v}$ ) is sufficient to place constraints on the orbital inclination, using only one radial-velocity curve: $i<43^{\circ}$. 
The results of test computations show that the variation in the radial-velocity curve due to variations in the orbital inclination $i$ grows with the mass of the optical star; this effect is greatest in binary systems with component-mass ratios $q<1$ (Tables 3-6). We can adopt the variations in the radial-velocity curves presented in Tables 3-6 to estimate the accuracy of observed radial-velocity curves required to reliably derive the orbital inclinations of observed binary systems. Inclination estimates are most feasible for X-ray binaries with massive optical components $\left(m_{v}>10 M_{\odot}\right.$ for systems whose relativistic component is a black hole with mass $m_{x} \simeq 10 M_{\odot}$ ). The required accuracy of the observed radial-velocity curve should be better than $4-8 \%$. Note that all our computations were carried out for a mass of the relativistic object of $m_{x}=10 M_{\odot}$. Since the variations in the radial-velocity curve are determined primarily by the ratio $q=m_{x} / m_{v}$, our main conclusions are also applicable for X-ray binary systems with neutron stars with masses $m_{x} \simeq 1.4 M_{\odot}$. In this case, the magnitude of the radial-velocity variations could be sufficient to estimate the orbital inclination if the mass of the optical star is $m_{v} \gtrsim 1 M_{\odot}$.

Recall that we have synthesized the radial-velocity curves using the $\mathrm{H} \gamma$ and $\mathrm{CaI} 6439 \AA$ absorption lines assuming LTE. It is known that the approximation of LTE is not suitable for hot stars with $T_{\text {eff }}>$ 20000-30000 K [11]. The inferred radial-velocity variations with variations in the orbital inclination could change when the radial-velocity curve is synthesized allowing for non-LTE corrections. However, note that our test computations using a constant profile shape for the $\mathrm{H} \gamma$ absorption line over the stellar surface (Figs. 1, 2) show that the variations in the shape of the integrated line profile are mainly sensitive to the geometry of the shape of the star (the position of the star's "nose" relative to the barycenter of the binary system). This provides hope that, although allowance for non-LTE effects could appreciably affect the equivalent widths of the $\mathrm{H} \gamma$ line in the case of high $T_{\text {eff }}$, the variations of the shape of the integrated $\mathrm{H} \gamma$ profile with orbital phase will differ only slightly with the use of LTE and non-LTE model atmospheres for the optical star. We plan to investigate this question further by carrying out corresponding computations in a non-LTE approximation.

\section{ACKNOWLEDGMENTS}

The authors thank L.I. Mashonkin for valuable comments. This work was supported by the Russian Foundation for Basic Research (project no. 02-0217524 ) and the program "Leading Scientific Schools of Russia” (grant no. NSh-388.2003.2).

\section{REFERENCES}

1. S. Sofia and R. E. Wilson, Astrophys. J. 203, 182 (1976).

2. É. A. Antokhina and A. M. Cherepashchuk, Pis'ma Astron. Zh. 23, 889 (1997) [Astron. Lett. 23, 773 (1997)].

3. T. Shahbaz, Mon. Not. R. Astron. Soc. 298, 153 (1998).

4. M. K. Abubekerov, É. A. Antokhina, and A. M. Cherepashchuk, Astron. Zh. 81, 606 (2004) [Astron. Rep. 48, 550 (2004)].

5. É. A. Antokhina, A. M. Cherepashchuk, and V. V. Shimanskii, Izv. Ross. Akad. Nauk, Ser. Fiz. 67 (3), 293 (2003).

6. É. A. Antokhina, A. M. Cherepashchuk, and V. V. Shimanskii, Astron. Zh. 82, 131 (2005) [Astron. Rep. 49, 109 (2005)].

7. M. K. Abubekerov, É. A. Antokhina, and A. M. Cherepashchuk, Astron. Zh. 81, 108 (2004) [Astron. Rep. 48, 89 (2004)].

8. É. A. Antokhina and A. M. Cherepashchuk, Astron. Zh. 71, 420 (1994) [Astron. Rep. 38, 367 (1994)].

9. R. L. Kurucz, CD-ROMs (Smithsonian Astrophysical Observatory, Cambridge, 1992).

10. A. M. Cherepashchuk, N. A. Katysheva, T. S. Khruzina, and C. Yu. Shugarov, Highly Evolved Close Binary Stars: Catalog (Gordon and Breach, New York, 1996), Vol. 1, Part 1, p. 82.

11. A. Herrero, IAU Symp. No. 212: A Massive Star Odyssei: from Main Sequence to Supernova, Ed. by Karel A. van der Hucht, Artemio Herrero, and Cesar Esteban (Astron. Soc. Pac., San Francisco, 2003), p. 3.

12. V. Straizhis, Metal-Deficient Stars (Mokslas, Vilnius, 1982) [in Russian].

13. É. A. Antokhina, Astron. Zh. 73, 532 (1996) [Astron. Rep. 40, 483(1996)].

14. N. I. Balog, A. V. Goncharskii, and A. M. Cherepashchuk, Pis'ma Astron. Zh. 7, 605 (1981) [Sov. Astron. Lett. 7, 336 (1981)].

Translated by D. Gabuzda 